\title{
Congenital Absence of the Pericardium
}

\author{
Lillian C. Man, MD, Jaehee Kim MD, Rakesh Gupta, MD
}

\section{INTRODUCTION}

Congenital absence of the pericardium (CAP) is an uncommon finding previously recognized only post-mortem or during surgery. However, its incidence has been on the rise with the use of multiple contemporary imaging techniques. CAP can present with paroxysmal, left-sided chest pain and should be considered in the differential diagnosis of atypical causes of chest pain. We present the case of a 50-year-old male who presented with chest pressure, symptoms of heart failure, and was found to be in atrial fibrillation in whom partial absence of the left pericardium was diagnosed.

\section{CASE PRESENTATION}

A 50 year-old African-American male presented to the Emergency Department with a chief complaint of shortness of breath (SOB) and worsening dyspnea on exertion for one week. He described worsening SOB when lying on his left side, as well as palpitations, orthopnea, and non-radiating, left-sided chest pressure. On physical exam, the patient was tachycardic with an irregularly irregular heart rate, crackles in the lower lobes, trace bilateral lower extremity edema, and a non-palpable apical impulse. Laboratory studies were significant for a pro-B-type natriuretic peptide (pro-BNP) level of $2830 \mathrm{pg} / \mathrm{mL}$ (normal range $=<100 \mathrm{pg} / \mathrm{mL}$ ) and negative troponins. Electrocardiogram (ECG) showed a ventricular rate of 146 beats per minute, irregularly irregular rhythm, right axis deviation, and poor R-wave progression. Chest X-ray (CXR) (Figure 1) revealed cardiomegaly and a diminished right heart border. Subsequent echocardiogram showed severe mitral regurgitation and moderate tricuspid regurgitation. Of note, it also showed the heart shifted posteriorly in the apical window, suspicious for congenital absence of pericardium. CT scan confirmed the diagnosis of partial absence of the left pericardium.

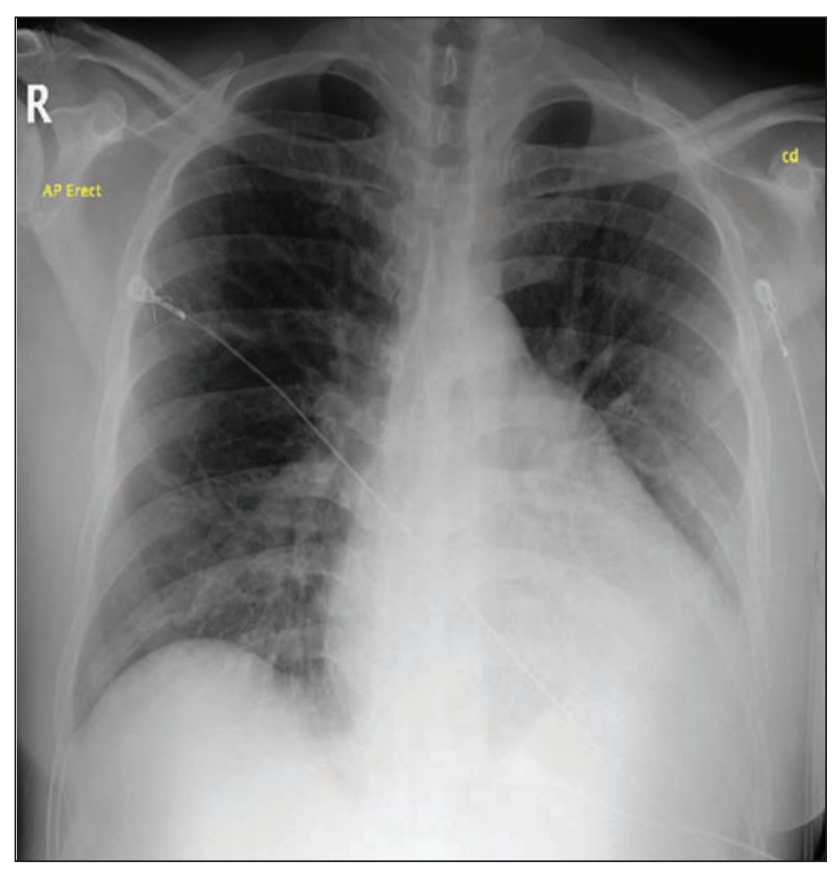

Figure 1: Admission chest X-ray

\section{DISCUSSION}

Congenital absence of the pericardium is a rare entity formerly recognized only at autopsy or during surgery ${ }^{1}$, with about 400 reported cases in the literature. Up to $70-80 \%$ of pericardial defects involve the left side and are due to premature atrophy of the left common cardinal vein during embryologic development. ${ }^{2}$ About one-third of all cases of CAP are described in association with other cardiac defects, such as patent ductus arteriosus, mitral stenosis, and tetralogy of Fallot. ${ }^{3.4}$

The clinical presentation of symptomatic patients tends to be non-specific. Patients with partial CAP may have dyspnea and trepopnea, or the presence of dyspnea when the lying on one side, which our patient had. ${ }^{4}$ A number of case reports also described patients who presented with left-sided chest pain of varying quality, 
such as stabbing, throbbing, postural, or exertional. ${ }^{3,5}$ In other case reports, patients have reported the sensation of a "shifting heart". Since the pericardium provides structural support for the heart, its complete or partial absence allows the cardiac apex to move posteriorly or laterally, leading to a significantly displaced or non-palpable apical impulse, ${ }^{3,4,6}$ which our patient had. Interestingly, patients with complete absence of the pericardium tend to be asymptomatic.

ECG findings may show right-axis deviation, right bundle branch block, and poor R-wave progression. ${ }^{2}$ These findings are related to the posterior displacement of the left ventricle, allowing the right heart to become more prominent anteriorly. CXR can show levoposition in which the heart is shifted laterally and into the left chest, leading to an absent right heart border. The left cardiac border may be straightened and elongated, known as the "Snoopy sign." Echocardiogram may show what appears to be a dilated right ventricle due to its anterior location and abnormal interventricular septal motion. ${ }^{5.78}$ CT or cardiac MRI are the best diagnostic tools for pericardial absence, with cardiac MRI being the gold standard?

Management depends on the size of the pericardial defect and presence or absence of symptoms. Patients with a partial pericardial absence are at risk for entrapment of the heart through the defect. This can lead to strangulation of the atria, appendages, and ventricles, causing sudden death in the most severe cases.79,10. Patients without such significant complications can still experience bothersome, sharp, left-sided chest pain. Surgical pericardioplasty is usually reserved for symptomatic patients or those with partial defects in which herniation is an imminent risk. ${ }^{2}$ On the other hand, patients with a large or complete pericardial defect can usually be safely observed because of a lower risk of herniation and incarceration of cardiac structures

\section{KEY POINTS}

Although CAP is usually an incidental finding, awareness of it is important for a number of reasons. Patients with partial defects may experience disabling, left-sided chest pain without obvious cause. In the most severe cases, the atria, appendages, or ventricles may herniate through the pericardial defect, leading to sudden death. Given that CAP has a nonspecific clinical presentation but the potential for severe complications, maintaining a high index of suspicion is important in diagnosing and managing this condition in symptomatic patients.

\section{REFERENCES}

1. Nasser WK, Helmen C, Tavel M, Feigenbaum H, Fisch C. Congenital absence of the left pericardium: clinical, electrocardiographic, radiographic, hemodynamic, and angiographic findings in six cases. Circulation. 1970; 41: 469-478.

2. Verde F, Johnson PT, Jha S, et al. Congenital absence of the pericardium and its mimics. Journal of Cardiovascular Computed Tomography. 2013; 7: 11-17.

3. Gatzoulis MA, Munk M, Merchant N, et al. Isolated congenital absence of the pericardium: clinical presentation, diagnosis, and management. Ann Thorac Surg. 2000; 69: 1209-1215

4. Abbas AE, Appleton CP, Liu PT, Sweeney JP. Congenital absence of the pericardium: case presentation and review of the literature. International Journal of Cardiology. 2005; 98: 21-25.

5. Garnier F, Eicher J, Philip J, et al. Congenital complete absence of the left pericardium: a rare cause of chest pain, pseudo-right heart overload. Clin Card. 2010; E52-E57.

6. Khandaker MH, Espinosa RE, Nishimura RA. Pericardial disease: diagnosis and management. Mayo Clinic Proc. 2010; 85(6): 572-593.

7. Brulotte S, Roy L, Larose E. Congenital absence of the pericardium presenting as acute myocardial necrosis. Can J Cardiol. 2007; 23(11): 909-912.

8. Montaudon M, Roubertie F, Bire F, Laurent F. Congenital pericardial defect: report of two cases and literature review. Surg Radiol Anat. 2007; 29: 195-200

9. Chassaing S, Bensouda C, Bar O, Barbey C, Blanchard D. A case of partial congenital absence of pericardium revealed by MRI. Circ Cardiovasc Imaging. 2010; 3; 632-634.

10. Jones JW, McManus BM. Fatal cardiac strangulation by congenital partial pericardial defect. Am Heart J. 1984; 107: 183-5. 